\title{
INTERAÇÕES MEDICAMENTOSAS POTENCIAIS E POLIFARMÁCIA EM PRESCRIÇÕES DE PACIENTES ACOMPANHADOS POR FARMACÊUTICOS CLÍNICOS EM UNIDADES DE TERAPIA INTENSIVA
}

\section{POTENTIAL DRUG INTERACTIONS AND POL YPHARMACY IN PRESCRIPTIONS OF PATIENTS ACCOMPANIED BY CLINICAL PHARMACISTS IN INTENSIVE CARE UNITS}

Beatriz da Silva Santos ${ }^{1}$, Tamiles Daiane Borges Santana², Ana Mércia Silva Mascarenhas ${ }^{3}$, Manoela dos Santos Silva $^{4}$, Alaine Azevedo Barbosa ${ }^{5}$, Bianca Oliveira Souza ${ }^{6}$, Danilo Bomfim Miranda ${ }^{7}$, Dara Evinny Santos de Oliveira ${ }^{8}$, Gisele da Silveira Lemos $^{9}$

Submetido em: 25/08/2021

Aprovado em: 01/10/2021 e29674

https://doi.org/10.47820/recima21.v2i9.674

\section{RESUMO}

A interação medicamentosa potencial (IMP) ocorre quando um medicamento modifica a intensidade dos efeitos farmacológicos de outro, aumentando ou diminuindo o resultado esperado da farmacoterapia, sendo sua ocorrência mais frequente na presença de polifarmácia. Este estudo teve como objetivo avaliar a presença de polifarmácia e IMP em prescrições de pacientes, acompanhados por farmacêuticos clínicos, internados em Unidade de Terapia Intensiva. Trata-se de um estudo transversal, descritivo, realizado em hospital público de referência regional com coleta referente ao período de julho a dezembro de 2019. Foram analisadas 102 prescrições, com um total de 1717 medicamentos, média de 16,8 medicamentos por prescrição. Observou-se 100\% de polifarmácia, sendo 97,1\% polifarmácia excessiva. Identificou-se 913 IMP, apresentando uma mediana de 7 IMP por prescrição. Quanto aos fármacos de maior frequência em IMP prevaleceram os subgrupos Fenilpiperidina $11,2 \%$, Benzodiazepina $11 \%$, Propulsivos 10,6\% e Insulinas e análogos para injeção, ação rápida 6,9\%, com envolvimento dos seguintes fármacos: Fentanila, Midazolam, Metoclopramida e Insulina Regular Humana. No que diz respeito às características sociodemográficas e clínicas dos pacientes, houve predomínio de sexo masculino $(52,5 \%)$, com idade $>60$ anos $(55,4 \%)$, raça parda $(94,0 \%)$. Com relação a comorbidades prevaleceu a Hipertensão Arterial Sistêmica (67, 8\%) e Diabetes Mellitus (39,0\%). Foi possível identificar um grande número de medicamentos prescritos, com presença da polifarmácia excessiva e uma frequência elevada de IMP, isso mostra que monitorar os pacientes em UTI é importante e necessário, e deve ser realizado por equipe multiprofissional, com inclusão do Farmacêutico clínico.

PALAVRAS-CHAVE: Polimedicação. Prescrição de Medicamentos. Interações Medicamentosas

\section{ABSTRACT}

Potential drug interaction (PMI) occurs when one drug modifies the intensity of the pharmacological effects of another, increasing or decreasing the expected result of pharmacotherapy, and its occurrence is more frequent in the presence of polypharmacy. This study aimed to evaluate the presence of polypharmacy and IMP in prescriptions for patients, accompanied by clinical pharmacists, admitted to the Intensive Care Unit. This is a cross-sectional, descriptive study, carried out in a public hospital of regional reference, with collection for the period from July to December 2019. 102 prescriptions were analyzed, with a total of 1717 medications, an average of 16.8 prescription drugs. 100\% polypharmacy was

${ }^{1}$ Curso de Graduação em Farmácia. Universidade Estadual do Sudoeste da Bahia.

2 Programa de Residência Multiprofissional em Urgência e Emergência. Universidade Estadual do Sudoeste da Bahia.

3 Programa de Residência em Urgência e Emergência. Universidade Estadual do Sudoeste da Bahia.

${ }^{4}$ Curso de Graduação em Farmácia. Universidade Estadual do Sudoeste da Bahia.

${ }^{5}$ Curso de Graduação em Farmácia. Universidade Estadual do Sudoeste da Bahia.

${ }^{6}$ Curso de Graduação em Farmácia. Universidade Estadual do Sudoeste da Bahia.

7 Curso de Graduação em Farmácia. Universidade Estadual do Sudoeste da Bahia.

${ }^{8}$ Curso de Graduação em Farmácia. Universidade Estadual do Sudoeste da Bahia.

9 Universidade Estadual do Sudoeste da Bahia

RECIMA21 - Ciências Exatas e da Terra, Sociais, da Saúde, Humanas e Engenharia/Tecnologia 


\section{RECIMA21 - REVISTA CIENTÍFICA MULTIDISCIPLINAR ISSN 2675-6218}

INTERAÇÕES MEDICAMENTOSAS POTENCIAIS E POLIFARMÁCIA EM PRESCRIÇÕES DE PACIENTES ACOMPANHADOS POR FARMACÊUTICOS CLÍNICOS EM UNIDADES DE TERAPIA INTENSIVA Beatriz da Silva Santos, Tamiles Daiane Borges Santana, Ana Mércia Silva Mascarenhas, Manoela dos Santos Silva, Alaine Azevedo Barbosa, Bianca Oliveira Souza,

observed, with $97.1 \%$ being excessive polypharmacy. 913 IMPs were identified, with a median of 7 IMPS per prescription. As for the most frequent drugs in IMP, the subgroups were $11.2 \%$ Phenylpiperidine, $11 \%$ Benzodiazepine, 10.6\% Propulsives and Insulins and analogues for injection, fast action 6.9\%, with involvement of the following drugs: Fentanyl, Midazolam, Metoclopramide and Human Regular Insulin. With regard to sociodemographic and clinical characteristics of patients, there was a predominance of males (52.5\%), aged $>60$ years (55.4\%), and mixed race (94.0\%). Regarding comorbidities, Systemic Arterial Hypertension (67.8\%) and Diabetes Mellitus (39.0\%) prevailed. It was possible to identify a large number of prescription drugs, with the presence of excessive polypharmacy and a high frequency of IMP, which shows that monitoring patients in the ICU is important and necessary, and should be performed by a multidisciplinary team, including the clinical pharmacist.

KEYWORDS: Polypharmacy. Drug Prescriptions. Drug Interactions

\section{INTRODUÇÃO}

Nas últimas décadas a preocupação com a segurança no uso dos medicamentos e a prevenção de interações medicamentosas (IM) na saúde dos pacientes tem despertado muita atenção dos pesquisadores ${ }^{1}$. Isso porque na prática clínica, o uso de vários medicamentos como medida de suporte terapêutico para tentar reestabelecer o equilíbrio fisiológico é comum no tratamento de várias doenças, o que expõe os pacientes a vários problemas relacionados a medicamentos (PRM), como por exemplo, as $\mathrm{IM}^{2}$.

A IM é uma a situação clínica que causa modificação, aumento ou diminuição do efeito de um fármaco diante de seu uso concomitante com outro medicamento, o que gera um desfecho terapêutico diferente daquele observado quando se administra apenas o medicamento isolado ${ }^{3}$. As IM são classificadas como potenciais, quando um medicamento apresenta a probabilidade de modificar a intensidade dos resultados farmacológicos de outro, e reais, quando se tem uma comprovação a partir da alteração clínica provocada pela ação de um fármaco sobre o outro².

Existem alguns fatores que contribuem para a ocorrência das IM, dentre elas temos o número de medicamentos prescritos, a duração da internação do paciente, a farmacoterapia, idade do paciente, estágio da doença, número de prescritores que o visitam o paciente, falta de conhecimento em relação às IM, e a falta de acompanhamento farmacêutico diário das interações. Esses fatores provocam um impacto negativo na segurança do paciente, uma vez que, elevam os custos de internamento e prolongam tempo de tratamento e aumentam a taxa de mortalidade ${ }^{1}$.

A IM é bastante comum no ambiente hospitalar, pois os pacientes são submetidos rotineiramente a práticas como infusão contínua de drogas vasoativas, e administração intermitente e essencial de outros fármacos ${ }^{2,4}$. Em estudo de revisão sistemática, foi observado que em 3.150 pacientes em unidade de terapia intensiva (UTI), 63\% eram expostos a pelo menos uma interação medicamentosa potencial $(\mathrm{IMP})^{5}$. As IM são frequentes na UTI, pois devido ao quadro clínico instável dos pacientes, esses apresentam absorção prejudicada, menor capacidade de eliminação do fármaco, diminuição da função renal e hepática e necessidade de polifarmácia ${ }^{6}$. 


\section{RECIMA21 - REVISTA CIENTÍFICA MULTIDISCIPLINAR ISSN 2675-6218}

INTERAÇÕES MEDICAMENTOSAS POTENCIAIS E POLIFARMÁCIA EM PRESCRIÇÕES DE PACIENTES ACOMPANHADOS POR FARMACÊUTICOS CLÍNICOS EM UNIDADES DE TERAPIA INTENSIVA Beatriz da Silva Santos, Tamiles Daiane Borges Santana, Ana Mércia Silva Mascarenhas, Danilo Bomfim Miranda, Dara Evinny Santos de Oliveira, Gisele da Silveira Lemos

A polifarmácia é definida como o uso concomitante de vários medicamentos, normalmente usando como critério valores de cinco ou mais fármacos. Embora esse valor seja o mais usado, existem diversas determinações numéricas para a polifarmácia, que varia de dois a onze medicamentos e dez ou mais medicamentos, sendo nesse último caso considerado por alguns autores como uma polifarmácia excessiva ${ }^{7}$.

Nesse contexto, percebe-se que há necessidade de ações colaborativas de profissionais farmacêuticos junto à equipe multiprofissional nas UTI para avaliar as prescrições e acompanhar os pacientes a beira leito, fazendo intervenções e monitorando manifestações clínicas das IM, mostrando os riscos e benefícios da farmacoterapia no paciente crítico, de forma a contribuir para um tratamento farmacológico apropriado, efetivo e seguro 2,8 .

Dessa forma, o objetivo desse estudo foi avaliar a presença de polifarmácia e interações medicamentosas potenciais em prescrições de pacientes, acompanhados por farmacêuticos clínicos, internados em unidades de terapia intensiva.

\section{METODOLOGIA}

\section{Tipo de estudo e local da pesquisa}

Trata-se de um estudo observacional, transversal, descritivo com abordagem qualitativa. As informações utilizadas para este estudo foram extraídas do banco de dados da pesquisa "Cuidado Farmacêutico: avaliação do uso de medicamentos em um hospital regional".

O estudo foi realizado em um hospital público localizado em um município na região Sudoeste da Bahia, que possui perfil de hospital porta-aberta de média e alta complexidade, tendo como principal foco o atendimento às Urgências e Emergências, e também referência em cirurgias ortopédicas. Possui 275 leitos, dentre os quais 29 são de UTI. Atualmente o hospital conta com 39 leitos para internamento intensivo, distribuídos em 4 UTI. Destes, 19 são direcionados para tratamento de pacientes com COVID19, 10 a pacientes cirúrgicos, 10 a pacientes com outras comorbidades. Atende à população de 26 municípios, sendo referência na região da Base Regional de Saúde Sul. Realiza atendimento em urgência e emergência, com classificação de risco de acordo com o protocolo estadual (azul, verde, amarelo e vermelho $)^{9}$ e também em clínica médica, neurologia clínica e cirúrgica, cirurgia geral e ortopedia, pediatria (urgência e emergência e enfermaria) e psiquiatria.

\section{Coleta de dados}

Os dados foram coletados por meio do preenchimento de formulário próprio elaborado para a pesquisa, tendo como fonte o prontuário do paciente. Foi realizado um teste piloto com o instrumento de coleta, no qual foram avaliados prontuários de dez pacientes, que não fizeram parte da amostra para este estudo. A pesquisa considerou o período de julho a dezembro de 2019, e a coleta dos dados foi realizada por equipe previamente treinada. 


\section{RECIMA21 - REVISTA CIENTÍFICA MULTIDISCIPLINAR ISSN 2675-6218}

INTERAÇÕES MEDICAMENTOSAS POTENCIAIS E POLIFARMÁCIA EM PRESCRIÇÕES DE PACIENTES ACOMPANHADOS POR FARMACÊUTICOS CLÍNICOS EM UNIDADES DE TERAPIA INTENSIVA Beatriz da Silva Santos, Tamiles Daiane Borges Santana, Ana Mércia Silva Mascarenhas, Danilo Bomfim Miranda, Dara Evinny Santos de Oliveira, Gisele da Silveira Lemos

\section{Amostra e critérios de inclusão e exclusão}

A amostra do estudo foi composta por pacientes admitidos nas UTI. Como critério de inclusão considerou-se a prescrição de 24 horas, após entrada do paciente nestas unidades, que foram acompanhados por farmacêuticos clínicos, e com idade superior a 18 anos. O diagrama do processo de inclusão dos pacientes no estudo encontra-se na Figura 1.

Figura 1- Diagrama do processo de inclusão dos pacientes no estudo. Bahia, Brasil, 2019.

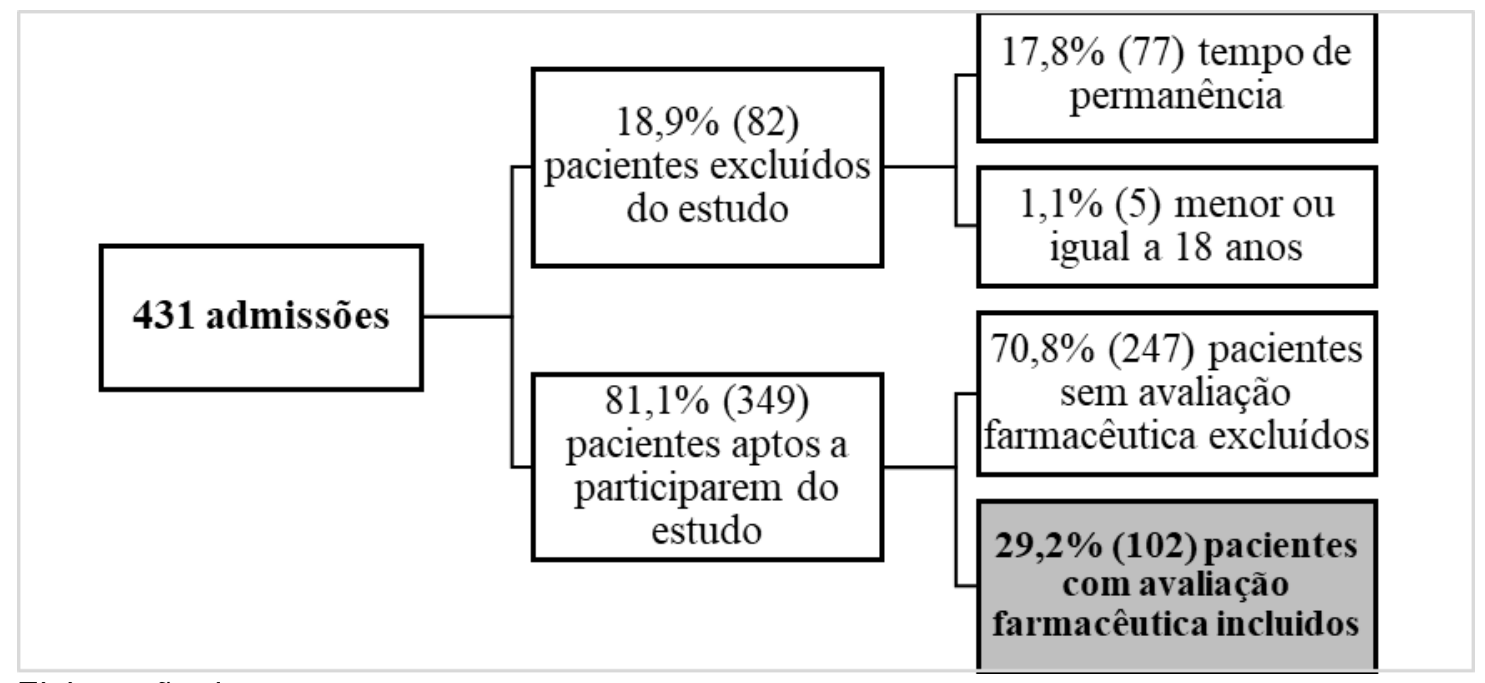

Elaboração dos autores

\section{Definições das variáveis}

\section{Variável dependente}

A presença de polifarmácia e IMP foram definidos e categorizadas em: Polifarmácia: $O$ uso de polifarmácia foi considerado como sim ou não e categorizado em uso de 5 - 9 medicamentos, polifarmácia, e o uso de 10 ou mais medicamentos, polifarmácia excessiva ${ }^{10}$. Ocorrência de IMP: Sim e não.

A partir dos dados da terapia farmacológica de cada paciente, foi então feita a avaliação das IMP por meio do aplicativo Micromedex Drug Int. ${ }^{\circledR}$, IBM Brasil, para Android, versão 4.0. Essa base de dados permite a análise de $\mathrm{IM}$, identificação de medicamentos, comparação entre medicamentos e calculadoras, sendo considerada como padrão de excelência em informação clínica ${ }^{11}$. As interações demonstradas por ele são classificadas de acordo com a sua gravidade (contraindicada, maior, moderada e menor) e documentação descritas no meio científico (excelente, boa e falha). A documentação falha se caracteriza quando a documentação disponível com evidência científica não é satisfatória 


\section{RECIMA21 - REVISTA CIENTÍFICA MULTIDISCIPLINAR ISSN 2675-6218}

INTERAÇÕES MEDICAMENTOSAS POTENCIAIS E POLIFARMÁCIA EM PRESCRIÇÕES DE PACIENTES ACOMPANHADOS POR FARMACÊUTICOS CLÍNICOS EM UNIDADES DE TERAPIA INTENSIVA Beatriz da Silva Santos, Tamiles Daiane Borges Santana, Ana Mércia Silva Mascarenhas, Danilo Bomfim Miranda, Dara Evinny Santos de Oliveira, Gisele da Silveira Lemos

Quanto a classificação dos medicamentos envolvidos em IMP, foi realizada através da Anatomical Therapeutic Chemical (ATC) da Organização Mundial da Saúde (OMS), que divide os princípios ativos em grupos de acordo com o órgão ou sistema onde eles atuam e de acordo com as suas propriedades terapêuticas, farmacológicas e químicas ${ }^{12}$.

\section{Variáveis independentes}

Foram considerados como variáveis independentes:

- Características sociodemográficas: Sexo (Feminino e masculino), Raça (branco e não branco), Faixa etária (19 a 59 anos e $\geq 60$ anos, idosos e não idosos, respectivamente) e Estado Civil (com companheiro e sem companheiro)

- Características clínicas: Hipertensão Arterial Sistêmica (HAS), Diabetes Mellitus (DM), Cardiopatia e Nefropatia: sim ou não.

\section{Análise dos dados}

Realizou-se análise descritiva, estimando-se as frequências e porcentagens absolutas e relativas das variáveis categóricas e medindo-se a tendência central (média e mediana) e a dispersão (desviopadrão e intervalo interquartílico) das variáveis quantitativas. Para avaliação da normalidade das variáveis numéricas foi utilizado o teste de Kolmogorov-Smirnov, considerando $p>0,05$, distribuição normal.

\section{Aspectos éticos}

A referida pesquisa atendeu todos os preceitos éticos. O estudo "Cuidado Farmacêutico: avaliação do uso de medicamentos em um hospital regional" foi aprovado pelo Comitê de Ética e Pesquisa (CEP) da UESB, protocolo ㄲo 4.229.023, CAAE 34826020.1.0000.0055.

\section{RESULTADOS}

A amostra do estudo foi composta por pacientes acompanhados por farmacêuticos clínicos, resultando num total de 102 pacientes, conforme critérios de inclusão. Com relação ao tempo de permanência dos pacientes na UTI verificou-se mediana de 13,5 dias com intervalo interquartil de 16,3. Dentre os pacientes incluídos no estudo, 32,4\% (33) foram internados na UTI 1, 28,4\% (29) na UTI 2, 9,8\% (10) na UTI 3, 25,5\% (26) passaram por 2 UTI no mesmo internamento e 3,9\% (4) passaram por 3 UTI.

Com relação ao sexo a maioria era do sexo masculino 52,5\% (53). A idade dos pacientes variou de 19 a 80 anos, com média de idade de 59,6 anos ( $D P=17,9$ ), sendo a faixa etária predominante $>60$ anos, idosos, com $55,4 \%$ (56). Quanto à raça, autorrelatada, observou-se uma maioria de pardos $94,0 \%$ 


\section{RECIMA21 - REVISTA CIENTÍFICA MULTIDISCIPLINAR ISSN 2675-6218}

INTERAÇÕES MEDICAMENTOSAS POTENCIAIS E POLIFARMÁCIA EM PRESCRIÇÕES DE PACIENTES ACOMPANHADOS POR FARMACÊUTICOS CLÍNICOS EM UNIDADES DE TERAPIA INTENSIVA Beatriz da Silva Santos, Tamiles Daiane Borges Santana, Ana Mércia Silva Mascarenhas, Danilo Bomfim Miranda, Dara Evinny Santos de Oliveira, Gisele da Silveira Lemos

(79). As comorbidades mais frequentes foram HAS 67, 8\% (59), DM 39,0\% (32), Cardiopatia 21,3\% (13) e Nefropatia 19,7\% (12), Tabela 1.

Foi identificado um total de 1717 medicamentos, sendo a média de medicamentos por prescrição de 16,8 (DP $=4,1)$. Foi identificado o uso de polifarmácia em $100,0 \%$ da amostra, sendo $97,1 \%$ do uso de 10 ou mais medicamentos, ou seja, polifarmácia excessiva.

Tabela 1 - Características sociodemográficas e clínicas de pacientes acompanhados por Farmacêuticos Clínicos internados em Unidades de Terapia Intensiva julho a dezembro de 2019. Bahia, Brasil. (N=102)

\begin{tabular}{|c|c|c|}
\hline Características sociodemográficas & $\mathbf{N}$ & $\%$ \\
\hline \multicolumn{3}{|l|}{$\operatorname{Sexo}(n=101)$} \\
\hline Feminino & 48 & 47,5 \\
\hline Masculino & 53 & 52,5 \\
\hline \multicolumn{3}{|l|}{ Faixa etária $(n=101)$} \\
\hline 19 a 59 anos & 45 & 44,6 \\
\hline 60 anos ou mais & 56 & 55,4 \\
\hline \multicolumn{3}{|l|}{ Raça/Cor $(n=84)$} \\
\hline Branco & 0 & 0 \\
\hline Não branco (preto e pardo) & 84 & 100,0 \\
\hline \multicolumn{3}{|l|}{ Estado Civil $(n=84)$} \\
\hline Com companheiro & 25 & 29,8 \\
\hline Sem companheiro & 59 & 70,2 \\
\hline Condições de Saúde pré-existentes (sim) & $\mathbf{N}$ & $\%$ \\
\hline Etilismo $(n=51)$ & 13 & 25,5 \\
\hline Tabagismo $(n=43)$ & 8 & 18,6 \\
\hline Hipertensão Arterial Sistêmica $(n=87)$ & 59 & 67,8 \\
\hline Diabetes Mellitus ( $\mathrm{n}=82)$ & 32 & 39 \\
\hline Cardiopatia $(n=61)$ & 13 & 21,3 \\
\hline Nefropatia $(n=61)$ & 12 & 19,7 \\
\hline Terapias utilizadas durante internamento (sim, $n=102)$ & $\mathbf{N}$ & $\%$ \\
\hline Drogas vasoativas & 68 & 67,3 \\
\hline Sedação & 74 & 73,3 \\
\hline Antibioticoterapia & 96 & 94,1 \\
\hline Corticoterapia & 52 & 51 \\
\hline Profilaxia LAMG & 92 & 90,2 \\
\hline
\end{tabular}

Fonte: Elaboração dos autores. LAMG: lesão aguda de mucosa gástrica.

A frequência de IMP foi de $100 \%$, ou seja, em todas as prescrições analisadas. Com relação ao número de IMP por prescrição verificou-se uma mediana de 7 e intervalo interquartil de 7,3. Foram encontradas um total de $913 \mathrm{IM}$, sendo 219 IM diferentes. Essas foram classificadas de acordo com a sua severidade e sua documentação. Quanto à severidade das IMP encontradas 2,1\% (19) foram contraindicadas, 62,0\% (556) maior, 28,1\% (263) moderada e 7,1\% (65) menor. Já de acordo com a documentação 13,7\% (125) foram consideradas excelente, 22,3\% (204) boa e 64,0\% (584) falha.

Para apresentação das IMP optou-se por aquelas que tiveram maior frequência, Tabela 2. É possível observar que algumas IMP de maior frequência, embora demonstrassem severidade maior ou moderada, apresentaram documentação falha. Com isso foram consideradas as IM de severidade maior e moderada com documentação boa ou excelente. 


\section{RECIMA21 - REVISTA CIENTÍFICA MULTIDISCIPLINAR ISSN 2675-6218}

INTERAÇÕES MEDICAMENTOSAS POTENCIAIS E POLIFARMÁCIA EM PRESCRIÇÕES DE PACIENTES ACOMPANHADOS POR FARMACÊUTICOS CLÍNICOS EM UNIDADES DE TERAPIA INTENSIVA Beatriz da Silva Santos, Tamiles Daiane Borges Santana, Ana Mércia Silva Mascarenhas, Danilo Bomfim Miranda, Dara Evinny Santos de Oliveira, Gisele da Silveira Lemos

Os medicamentos envolvidos em IMP foram classificados de acordo com a classificação ATC, nível 4 (subgrupo terapêutico/farmacológico/químico), tabela 3. Observou-se que os subgrupos terapêuticos que obtiveram maior frequência em IMP foram os pertencentes aos subgrupos dos derivados de Fenilpiperidina 11,2\% (204), Benzodiazepina 11\% (201), Agentes Propulsivos 10,6\% (193), Insulinas e análogos para injeção, ação rápida 6,9\% (126), antagonistas do receptor H2 6,4\% (116), derivados de Hidantoína 6,2\% (113), e Outros Opióides 3,1\% (56), com envolvimento dos seguintes fármacos: Fentanila, Midazolam, Metoclopramida, Insulina Regular Humana, Ranitidina, Fenitoína e Tramadol. 


\section{RECIMA21 - REVISTA CIENTÍFICA MULTIDISCIPLINAR \\ ISSN 2675-6218}

INTERAÇÕES MEDICAMENTOSAS POTENCIAIS E POLIFARMÁCIA EM PRESCRICCÕES DE PACIENTES ACOMPANHADOS POR FARMACÊUTICOS CLINICOS EM UNIDADES DE TERAPIA INTENSIVA Beatriz da Silva Santos, Tamiles Daiane Borges Santana, Ana Mércia Silva Mascarenhas, Manoela dos Santos Silva, Alaine Azevedo Barbosa, Bianca Oliveira Souza,
Danilo Bomfim Miranda, Dara Evinny Santos de Oliveira, Gisele da Silveira Lemos

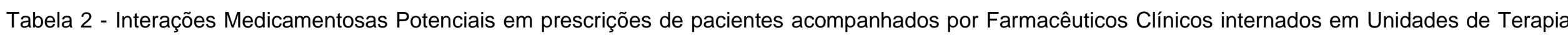
Intensiva. Bahia, Brasil, 2019. ( $\mathrm{N}=913)$

\begin{tabular}{|c|c|c|c|c|}
\hline IMP & Severidade & Doc. & Fr (\%) & Resultado \\
\hline Insulina NPH x Metoclopramida & Maior & Falha & 3,6 & Risco de hiperglicemia \\
\hline Fentanila x Midazolam & Maior & Falha & 3,3 & Aumento do risco de depressão do SNC. \\
\hline Metoclopramida x Midazolam & Maior & Falha & 2,4 & Aumento do risco de depressão do SNC. \\
\hline Fentanila x Metoclopramida & Maior & Falha & 2,2 & Aumento do risco de depressão do SNC \\
\hline Midazolam x Ranitidina & Moderada & Falha & 1,9 & Aumento da biodisponibilidade do midazolam \\
\hline Fentanila x Ranitidina & Maior & Excelente & 1,7 & Risco aumentado de toxicidade por fentanila \\
\hline Piperacilina $x$ vancomicina & Maior & Boa & 1,3 & Risco aumentado de lesão renal aguda \\
\hline Fenitoína x Ranitidina & Maior & Boa & 1,2 & Aumento das concentrações de fenitoína \\
\hline Midazolam x Omeprazol & Moderada & Falha & 1,2 & Toxicidade dos benzodiazepínicos (depressão do SNC, ataxia, letargia) \\
\hline AAS x Insulina Regular & Moderada & Falha & 1,0 & Aumento do risco de hipoglicemia \\
\hline Midazolam x Fenitoína & Moderada & Boa & 0,9 & Diminuição da eficácia do midazolam \\
\hline Fentanila x Fenitoína & Maior & Excelente & 0,8 & Diminuição das concentrações plasmáticas de fentanila. \\
\hline Fentanila x Ondasentrona & Maior & Falha & 0,8 & Risco aumentado de síndrome da serotonina. \\
\hline AAS x Clopidogrel & Maior & Falha & 0,7 & Risco aumentado de sangramento. \\
\hline Clopidogrel x Sinvastatina & Moderada & Excelente & 0,7 & $\begin{array}{c}\text { Diminuição da formação do metabólito ativo do clopidogrel, resultando em } \\
\text { alta reatividade plaquetária durante o tratamento. }\end{array}$ \\
\hline Ranitidina x Tramadol & Maior & Falha & 0,7 & Aumento da exposição ao tramadol do risco de depressão respiratória. \\
\hline Fentanila x Prednisona & Maior & Excelente & 0,6 & Diminuição das concentrações plasmáticas de fentanila. \\
\hline Metoclopramida x Tramadol & Maior & Falha & 0,6 & Aumento do risco de depressão do SNC. \\
\hline Outras & --- & --- & $74,4 \%$ & --- \\
\hline Total & --- & --- & 100,0 & -- \\
\hline
\end{tabular}

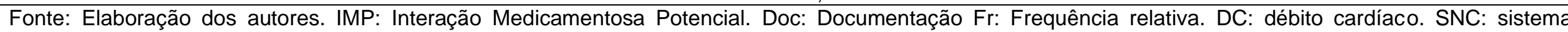
nervoso central. AAS: ácido acetilsalicílico 


\section{RECIMA21 - REVISTA CIENTÍFICA MULTIDISCIPLINAR ISSN 2675-6218}

INTERAÇÕES MEDICAMENTOSAS POTENCIAIS E POLIFARMÁCIA EM PRESCRIÇÕES DE PACIENTES ACOMPANHADOS POR FARMACÊUTICOS CLÍNICOS EM UNIDADES DE TERAPIA INTENSIVA Beatriz da Silva Santos, Tamiles Daiane Borges Santana, Ana Mércia Silva Mascarenhas, Danilo Bomfim Miranda, Dara Evinny Santos de Oliveira, Gisele da Silveira Lemos

Tabela 3 - Perfil de medicamentos envolvidos em interações medicamentosas potenciais, pela Anatomical Therapeutic Chemical, em prescrições de pacientes acompanhados por Farmacêuticos Clínicos internados em Unidades de Terapia Intensiva. Bahia, Brasil, 2019. ( $N=1717)$.

\begin{tabular}{cccc}
\hline & ATC Nível 4 & Medicamentos & Fr \% \\
\hline N02AB & Derivados de fenilpiperidina & Fentanila & 11,2 \\
N05CD & Derivados de benzodiazepina & Midazolam & 11 \\
A03FA & Propulsivos & Metoclopramida & 10,6 \\
A10AB & Insulinas e análogos para injeção, ação & Insulina Regular & 6,9 \\
A02BA & rápida. & Humana & 6,4 \\
N03AB & Antagonistas do receptor H2 & Ranitidina & 6,2 \\
B01AC & Derivados de hidantoína & Fenitoína & 4,5 \\
N02AX & Antitrombóticos- inibidores da agregação & AAS & 3,1 \\
B01AC & plaquetária excl. heparina & Tramadol & 2,9 \\
A02BC & Outros Opióides & Clopidogrel & 2,2 \\
C10AA & plaquetária excluindo heparina & Omeprazol & 2,2 \\
J01CR & Inibidores da bomba de prótons & Sinvastatina & 2 \\
J02AC & Combinaçares de penicilinas, & Piperacilina & 2 \\
J01XA & incl. inibidores de beta-lactamase & Fluconazol & 1,8 \\
A04AA & Derivados de triazole & Vancomicina & 1.8 \\
C03CA & Antibacterianos glicopepitídeos & Ondasentrona & 1,7 \\
H02AB & Sulfonamidas, simples & Furosemida & 1,6 \\
C01BD & Glicocorticoides & Prednisona & 1,4 \\
---- & Antiarrítmicos classe III & Amiodorona & 1,2 \\
--- & --- & Outros medicamentos & 14,3 \\
\hline
\end{tabular}

Fonte: Elaboração dos autores. ATC: Anatomica ITherapeutic Chemical. Fr: Frequência relativa

\section{DISCUSSÃO}

A presente pesquisa realizada com pacientes internados em UTI identificou a ocorrência de polifarmácia e IM em prescrições. Esse fato pode ser justificado, pois nessas unidades, os pacientes são submetidos a tratamentos com o uso de vários medicamentos, devido ao seu quadro clínico instável, o que favorecerá o surgimento de $\mathrm{IM}^{5}, 13$, podendo seus efeitos acontecerem de forma sinérgicas, potencializando o efeito terapêutico do fármaco, ou agir de forma antagônica, reduzindo a sua eficácia ${ }^{14}$.

O número de medicamentos prescritos por dia nas UTI corresponde a um dos indicadores de risco, pois há uma proporcionalidade direta entre o número de fármacos e o desenvolvimento de $\mathrm{IM}^{15}$. Além disso, existem outros fatores que também contribuem para o surgimento de uma IMP, como os fatores relacionados ao paciente, idade e comorbidades, aos medicamentos em uso, a dose prescrita, uso de drogas potenciais inibidores ou indutores de enzimas e margem terapêutica estreita, ao número de profissionais de saúde que cuidam do mesmo paciente e ao tempo de internação ${ }^{6,14,16}$.

Nesse estudo foram avaliadas as prescrições de pacientes acompanhados por farmacêuticos clínicos na UTI, sendo o tempo de internamento semelhante ao encontrado nos estudos de Rodrigues et 


\section{RECIMA21 - REVISTA CIENTÍFICA MULTIDISCIPLINAR ISSN 2675-6218}

INTERAÇÕES MEDICAMENTOSAS POTENCIAIS E POLIFARMÁCIA EM PRESCRIÇÕES DE PACIENTES ACOMPANHADOS POR FARMACÊUTICOS CLÍNICOS EM UNIDADES DE TERAPIA INTENSIVA Beatriz da Silva Santos, Tamiles Daiane Borges Santana, Ana Mércia Silva Mascarenhas, Danilo Bomfim Miranda, Dara Evinny Santos de Oliveira, Gisele da Silveira Lemos

al. ${ }^{17}$, que avaliaram a prevalência de IMP em prescrições de paciente de UTI de um hospital universitário do sistema público de saúde brasileiro, com mediana de 13,3 dias. Esses autores discorrem que, embora exista uma correlação entre o tempo de internamento e surgimento de IMP, não é tão claro se essas causam aumento no tempo de permanência do paciente na UTI ou se o período prolongado causa IMP. Além disso, justifica que o aumento no número de IMP em pacientes internados por longos períodos na UTI está relacionado à necessidade de uma terapia farmacológica com elevado número de medicamentos ${ }^{17}$.

A faixa etária predominante no estudo foi de pacientes idosos, maiores 60 anos, e do sexo masculino. Esse resultado corrobora com o encontrado por Silva et al. ${ }^{13}$, que buscou identificar e classificar as principais IMP e intervenções farmacêuticas observadas nas UTI de um hospital privado em Macapá (AP), com uma média de idade 61,14 anos, e prevalência do sexo masculino. Quanto à raça, houve um predomínio de pacientes não brancos, sendo esse resultado superior ao encontrado em outro trabalho que avaliou a influência de fatores sociodemográficos e clínicos na ocorrência de IMP e de eventos adversos a medicamentos ${ }^{2}$.

Pacientes idosos normalmente apresentam uma maior probabilidade de fazerem uso da polifarmácia, bem como tem suscetibilidade de sofrer IMP e reações adversas, isso devido ao fato de os idosos apresentarem um número elevado de comorbidades, o que influencia diretamente no uso de medicamentos ${ }^{18}$, eles também sofrem alterações fisiológicas como redução da acidez gástrica, água corporal e albumina sérica, aumento na percentagem de gordura corporal, diminuição de volume de distribuição de fármacos hidrossolúveis, diminuição de fluxo sanguíneo hepático, entre outros, tornandoos mais propensos a terem reações adversas a medicamentos ${ }^{18}$.

No que diz respeito à prevalência do sexo masculino, neste estudo, os dados corroboram com o encontrado por Sousa et al. ${ }^{19}$ que analisou as IMP em pacientes internados na UTI adulta em um hospital de ensino na cidade de Sobral, o qual justificativa que os homens geralmente são mais invulneráveis, o que os tornam propensos ao desleixo com o autocuidado, além disso, eles são mais expostos a situações de risco, ou seja, geralmente as agressões sofridas por eles são mais graves necessitando de um maior tempo de internação ${ }^{19}$.

Com relação à presença de comorbidades, as mais frequentes foram HAS e DM. No presente estudo, a frequência dessas condições de saúde foi inferior à encontrada por Dias et al. ${ }^{20}$, na avaliação de IMP, na alta hospitalar em idosos, com $71 \%$ de paciente hipertensos e $43,1 \%$ com diabetes, o uso associado de vários fármacos para tratar essas doenças, podem justificar a presença dessas IMP.

Com relação as terapias utilizadas pelos pacientes as drogas vasoativas (DVA) estão entre as mais usadas em unidades de terapia intensiva. As DVA são fármacos que visam corrigir a instabilidade hemodinâmica, auxiliando na melhora terapêutica dos pacientes. É de extrema importância estar atento a sua administração, pois quando inadequada podem ocasionar efeitos colaterais graves e, algumas vezes, irreversíveis ${ }^{21}$. 


\section{RECIMA21 - REVISTA CIENTÍFICA MULTIDISCIPLINAR ISSN 2675-6218}

INTERAÇÕES MEDICAMENTOSAS POTENCIAIS E POLIFARMÁCIA EM PRESCRIÇÕES DE PACIENTES ACOMPANHADOS POR FARMACÊUTICOS CLÍNICOS EM UNIDADES DE TERAPIA INTENSIVA Beatriz da Silva Santos, Tamiles Daiane Borges Santana, Ana Mércia Silva Mascarenhas, Danilo Bomfim Miranda, Dara Evinny Santos de Oliveira, Gisele da Silveira Lemos

O uso de antibióticos (ATB) é frequente em UTI, estima-se que cerca de $70 \%$ dos pacientes internados nessas unidades receberão ATB para tratar algum tipo de infecção ${ }^{22}$, justificando os valores encontrados no presente estudo. O uso indiscriminado de ATB pode favorecer a resistência bacteriana, uma vez que cepas com mecanismos de resistência serão selecionadas, diminuindo a oferta de opções terapêuticas ${ }^{23}$.

Já o uso de corticosteróides em UTI, está associado ao tratamento adjuvante das infecções, com objetivo de atenuar a resposta inflamatória local e sistêmica, sendo seu uso frequente em pacientes graves $^{24}$. Outra justificativa se deve ao fato desses fármacos modularem o processo inflamatório, regulando a expressão de vários genes inflamatórios e imunológicos ${ }^{25}$.

No que se refere à polifarmácia, verificou-se uma média elevada de medicamentos por prescrição, indicando a presença não só de polifarmácia, mais também de uma polifarmácia excessiva ${ }^{7}$. Estudo realizado em um hospital de ensino no interior do Rio Grande do Sul avaliou que em 308 prescrições, 101 apresentaram pelo menos uma IM, resultando em uma média de 13,3 medicamentos por prescrição, sendo esse resultado menor do que o valor encontrado nesse estudo ${ }^{26}$. Desta forma, destaca-se a necessidade de reflexão sobre as práticas de prescrição adotadas neste ambiente, assim como a necessidade de gestão da farmacoterapia por profissionais qualificados para tal.

A frequência de IMP encontrada no presente estudo mostrou-se elevada quando comparado ao estudo de Mehralian et al. ${ }^{1}$, que avaliaram as IM benéficas e prejudiciais em pacientes internados em UTI, em que $60,5 \%$ tinham pelo menos uma IM em seu perfil, sendo um total de 663 IM detectadas, com 574 prejudiciais e o restante benéfico, mostrando que a prevalência de IM é alta na UTI, e a maioria é prejudicial $(86 \%)$.

Neste estudo optou-se por discutir as IM de severidade maior e moderada, com documentação boa ou excelente. Os resultados para a severidade das IMP encontradas neste estudo estão de acordo com o relatado por Dutra et al. ${ }^{4}$ que obtiveram 2,09\% contraindicada, 63,37\% maior, 33,60\% moderada, tendo apenas diferença na severidade menor, que apresentou resultado de 0,94\%, sendo esse inferior. Já quanto à documentação, os resultados obtidos apresentaram-se próximos ao encontrado por Santos ${ }^{2}$, com $13,4 \%$ excelente, $31,4 \%$ boa e $51,4 \%$ falha.

Embora a IM entre Fentanila e Midazolam apresentasse documentação falha, as evidências científicas encontradas demonstram que ela é uma IM muito frequente e de grande relevância, sendo essa também levada em consideração. Mesmo sendo utilizada para fins terapêuticos, essa IM pode estar associada a eventos de sedação excessiva e hipotensão pelo seu uso inadequado². É bastante comum o uso dessa terapia em UTI por promoverem conforto e redução da ansiedade em pacientes em ventilação mecânica, e melhorar a oxigenação $2,27$.

A IM entre fentanila e ranitidina apresenta severidade maior, e resulta no aumento da toxicidade por fentanila ${ }^{28}$. A Ranitidina é inibidora do sistema enzimático dos opióides, o que favorece um aumento da biodisponibilidade e da toxicidade dos mesmos, podendo aumentar os efeitos secundários da fentanila causando depressão respiratória ${ }^{14}$. 


\section{RECIMA21 - REVISTA CIENTÍFICA MULTIDISCIPLINAR ISSN 2675-6218}

INTERAÇÕES MEDICAMENTOSAS POTENCIAIS E POLIFARMÁCIA EM PRESCRIÇÕES DE PACIENTES ACOMPANHADOS POR FARMACÊUTICOS CLÍNICOS EM UNIDADES DE TERAPIA INTENSIVA Beatriz da Silva Santos, Tamiles Daiane Borges Santana, Ana Mércia Silva Mascarenhas, Danilo Bomfim Miranda, Dara Evinny Santos de Oliveira, Gisele da Silveira Lemos

A piperacilina + tazobactam com a vancomicina foi uma IM frequente, apresentando resultado superior ao encontrado em outro estudo com 0,07\% ${ }^{4}$. Essa IM tem elevada severidade, podendo provocar aumento do risco de lesão renal aguda (LRA) quando usados concomitantemente ${ }^{29}$. Um estudo realizado por Alvarez ${ }^{30}$ avaliou a nefrotoxicidade de antimicrobianos e mostrou que o uso combinado de algumas penicilinas, como a piperacilina + tazobactam, com a vancomicina pode aumentar significativamente a incidência de LRA, sendo esse aumento 2 a 3 vezes maior quando comparado ao uso isolado da vancomicina.

A IM entre a fenitoína e ranitidina classifica-se com severidade maior, podendo provocar aumento das concentrações séricas de fenitoína ${ }^{31}$. Conforme estudo realizado por Silva et al. ${ }^{32}$, a ranitidina, por meio do seu estímulo metabólico, reduz os níveis séricos de fenitoína, diminuindo sua eficácia terapêutica, o que aumenta os riscos de crise convulsivas. A frequência encontrada no presente estudo se mostrou menor quando comparada a pesquisa realizada em três UTI do Hospital Universitário da Universidade Estadual de Londrina, com 5,5\%².

A frequência da IM entre fentanila e fenitoína demonstrou-se inferior quando comparada aos estudos de Silva e Damascena ${ }^{15}$, com $13 \%$. Essa IM, pode resultar em diminuição dos níveis plasmáticos da fentanila, causada pela indução do seu metabolismo, provocando redução de sua ação. Quando necessário, o ajuste de dose ou mudança na terapia devem ser realizados ${ }^{33}$. Outra IM envolvendo a fenitoína pode acontecer com o midazolam, com risco de diminuição da eficácia desse ${ }^{34}$. Essa redução é evidenciada clinicamente somente na primeira administração quando por via oral e pode ser realizado ajuste de dose pelo prescritor².

A associação entre clopidogrel e sinvastatina pode causar diminuição da formação do metabólito ativo do clopidogrel, provocando alta reatividade plaquetária durante o tratamento ${ }^{35}$. A frequência dessa IM no presente estudo foi menor, quando comparado ao encontrado por Dutra et al. ${ }^{4}$. A sinvastatina pode alterar a forma como o clopidogrel atua no organismo, interferindo no seu mecanismo de ação e diminuindo a sua eficácia. É necessário monitorar os pacientes em uso dessa estatina, podendo sugerir a troca por pravastatina, uma vez que, esse fármaco não interfere na forma como o clopidogrel se torna ativo no organismo ${ }^{36}$.

No caso da IM da fentanila e prednisona pode haver uma diminuição das concentrações plasmáticas da fentanila, pois a indução do seu metabolismo é mediada pela prednisona ${ }^{37}$. Corresponde a uma IM de severidade maior, e com frequência superior quando comparada com a literatura, que apresentou $0,21 \%^{4}$. Essa é uma associação que deve ser realizada com cuidado, sendo necessário o acompanhamento do paciente quanto aos sinais de abstinência dos opióides, pois quando for realizada a retirada da prednisona os níveis de fentanila podem aumentar de tal forma que poderá causar efeitos adversos, como a depressão respiratória, nesse sentido, o ajuste de dose pode ser realizado ${ }^{50}$.

Com base na análise das IMP deste estudo, foi possível identificar os fármacos mais frequentes envolvidos em IM (Fentanila, Midazolam, Metoclopramida, Insulina Regular Humana, Ranitidina, Fenitoína e Tramadol), que juntamente com a polifarmácia excessiva demonstram a complexidade do 


\section{RECIMA21 - REVISTA CIENTÍFICA MULTIDISCIPLINAR ISSN 2675-6218}

INTERAÇÕES MEDICAMENTOSAS POTENCIAIS E POLIFARMÁCIA EM PRESCRIÇÕES DE PACIENTES ACOMPANHADOS POR FARMACÊUTICOS CLÍNICOS EM UNIDADES DE TERAPIA INTENSIVA Beatriz da Silva Santos, Tamiles Daiane Borges Santana, Ana Mércia Silva Mascarenhas, Danilo Bomfim Miranda, Dara Evinny Santos de Oliveira, Gisele da Silveira Lemos

cuidado $^{2,38}$. Assim, se faz necessário a gestão das prováveis interações entre medicamentos em uso pelo paciente, com o objetivo de se conhecer os riscos, benefícios e manejo clínico, evitando o aparecimento de eventos adversos, os quais muitas vezes afetam a saúde do paciente podendo até mesmo ser fatal ${ }^{15}$.

A Fentanila e o Midazolam são medicamentos envolvidos em IM com alta frequência. Embora apresentem IM entre eles, esses fármacos são bastante prescritos em UTI e essa interação é por vezes intencional para promover a sedação dos pacientes que se encontram em ventilação mecânica, por exemplo $2,3,39$.

No que diz respeito ao uso de sedação, percebe-se que a sua frequência pode estar relacionada às frequências obtidas para os fármacos fentanila e midazolam, o que está de acordo com estudo realizado em UTI, no qual a fentanila e o midazolam apresentaram $14,5 \%$ das IM identificadas, estando envolvidos em 9 das $15 \mathrm{IM}$ mais frequentes ${ }^{39}$.

A Metoclopramida é frequentemente utilizada em UTI para o tratamento de desordens gastrintestinais a fim de evitar eventos como o refluxo gastroesofágico ${ }^{13}$. As IM envolvendo esses fármacos podem estar relacionadas à sua capacidade em estimular o esvaziamento gástrico ou provocar aumento na velocidade de absorção de muitos fármacos ${ }^{40}$.

Outro fármaco frequente em IM foi a Insulina Regular Humana, um hormônio produzido pelas células $\beta$ do pâncreas, e utilizada para o controle glicêmico de pacientes internados em UTI, de forma constante, o que justifica o seu amplo uso nesses locais ${ }^{13}$.

Já a ranitidina, embora tenha apresentado frequência nas UTI, seu uso foi restringido no mercado, após a ANVISA proibir, de forma definitiva, desde a comercialização até a propaganda o fármaco cloridrato de ranitidina, devido detecção de substâncias de N-nitrosodimetilamina (NDMA), nos medicamentos com o insumo farmacêutico ativo do cloridrato de ranitidina ${ }^{41}$.

A fenitoína é um fármaco frequente em prescrições de UTI, podendo ser usada em associação com benzodiazepínicos, devido a sua ação curta ou como droga de escolha quando esses não são suficientes para controlar as crises convulsivas ${ }^{42}$. As características desse medicamento, que podem favorecer as IMP podem estar ligadas ao seu índice terapêutico estreito e sua potente indução enzimática o que gera consequências clínicas importantes ${ }^{43}$.

O Tramadol, um analgésico opióide sintético de ação central, é um dos fármacos mais prescrito em UTI, por produzir analgesia causando alívio da resposta ao estresse e diminuindo complicações em pacientes críticos ${ }^{13}$. É considerado um fármaco de alta vigilância ou também chamado de medicamentos potencialmente perigosos (MPP), que apresenta alto risco de provocar danos significativos quando utilizadas de maneira errada ${ }^{39}$. Apesar de ter uso habitual em UTI, ainda é pouco reconhecido como um MPP. Em um estudo que investigou o conhecimento dos profissionais de enfermagem e farmacêuticos em relação à identificação de MPP, o tramadol, era utilizado por $99 \%$ dos entrevistados e assumido como MPP por apenas $46 \%$ destes $^{43}$. 


\section{RECIMA21 - REVISTA CIENTÍFICA MULTIDISCIPLINAR ISSN 2675-6218}

INTERAÇÕES MEDICAMENTOSAS POTENCIAIS E POLIFARMÁCIA EM PRESCRIÇÕES DE PACIENTES ACOMPANHADOS POR FARMACÊUTICOS CLÍNICOS EM UNIDADES DE TERAPIA INTENSIVA Beatriz da Silva Santos, Tamiles Daiane Borges Santana, Ana Mércia Silva Mascarenhas, Danilo Bomfim Miranda, Dara Evinny Santos de Oliveira, Gisele da Silveira Lemos

Nas UTIs os farmacêuticos clínicos se encontram aptos a acompanhar os pacientes em todo o processo de prescrição, preparação, dispensação, administração, e acompanhamento da utilização de medicamentos, como forma de intervenção farmacêutica ${ }^{44}$. Um estudo feito por Magalhães et al. ${ }^{45}$, analisou a contribuição do farmacêutico clínico nos serviços de saúde após a implantação do serviço de farmácia clínica na UTI, o resultado foi de 371 intervenções farmacêuticas, onde 202 foram comunicadas direto com à equipe médica, com 106 aceitas e 96 não aceitas. Isso mostra que a inclusão desse profissional junto à equipe multiprofissional contribui para melhoria dos desfechos clínicos.

Ainda nesse estudo, Magalhães et al. ${ }^{45}$, trazem que durante um período de 4 a 5 meses, os custos com a saúde em uma instituição sem a presença do farmacêutico clínico na UTI, acresceu de 209.000 a 280.000 dólares. Todavia, quando observados esses valores após a inserção deste profissional na UTI, a maior parte dos custos pôde ser evitada devido às intervenções feitas em participação em rounds e em revisão de prontuários.

O presente estudo apresentou como limitações a avaliação de um curto período de tempo do paciente em UTI, o que pode não levar ao real conhecimento de mais interações medicamentosas ou a polifarmácia excessiva.

Devido ao fato de os pacientes internados nas UTI receberem uma ampla gama de medicamentos, é perceptível a importância de se estudar a presença de IMP em prescrições de pacientes nesse ambiente, pois isso ajuda a reduzir os riscos e os eventos adversos evitáveis, garantindo uma melhor segurança para a saúde dos pacientes. E essa pesquisa traz informações importantes a respeito do uso da polifarmácia em UTI, bem como a frequência e riscos das IMP em pacientes nessas unidades, servindo de embasamento no processo de monitorização e acompanhamento pela equipe de saúde

\section{CONSIDERAÇÕES FINAIS}

Esse trabalho avaliou as IMP em prescrições de pacientes acompanhados por farmacêuticos clínicos em UTI, onde foi possível identificar um grande número de medicamentos prescritos, caracterizando a polifarmácia, também uma frequência elevada de interações medicamentosas, o que demonstra alguns dos possíveis PRM que os pacientes críticos podem estar sofrendo.

Isso nos mostra que o monitoramento dos pacientes em UTI é importante e necessário, e deve ser realizado por equipe multiprofissional, com inclusão do Farmacêutico clínico, que detém das informações a respeito dos medicamentos, garantindo uma prescrição mais segura, com menores riscos para a saúde dos pacientes. 


\section{RECIMA21 - REVISTA CIENTÍFICA MULTIDISCIPLINAR ISSN 2675-6218}

INTERAÇÕES MEDICAMENTOSAS POTENCIAIS E POLIFARMÁCIA EM PRESCRIÇÕES DE PACIENTES ACOMPANHADOS POR FARMACÊUTICOS CLÍNICOS EM UNIDADES DE TERAPIA INTENSIVA Beatriz da Silva Santos, Tamiles Daiane Borges Santana, Ana Mércia Silva Mascarenhas, Danilo Bomfim Miranda, Dara Evinny Santos de Oliveira, Gisele da Silveira Lemos

\section{REFERÊNCIAS}

1 Mehralian HA, Moghaddasi J, Rafiei $\mathrm{H}$. The prevalence of potentially beneficial and harmful drugdrug interactions in intensive care units. Drug metabolism and personalized therapy. 2019;34(1).

2 Santos MHBA. Análise de interações medicamentosas potenciais e de eventos adversos a medicamentos em uma unidade de terapia intensiva. [Mestrado em Epidemiologia em Saúde Pública]. Rio de Janeiro: Fundação Oswaldo Cruz; 2017.

3 Teixeira LHS, Maximo MP, Vieira ARM, Souza LNF, Batista AD, Fonseca CSM. Interações medicamentosas em unidades de terapia intensiva do Brasil: Revisão integrativa. Brazilian Journal of Health Review. 2021;4(2):7782-96.

4 Dutra AP, Lemos LM, Damascena RS. Avaliação do Perfil das Interações Medicamentosas e os Fatores Associados em Prescrições Médicas de Pacientes Internados em Unidade de Terapia Intensiva. Revista de psicologia. 2019;13(43):543-58.

5 Pessoa TD, Clemente Junior WS, Costa TX, Bezerra PK, Martins RR. Interações medicamentosas em terapia intensiva materna: prevalência, fatores e medicamentos de risco. Einstein. $2019 ; 17(3)$.

6 Bakker T, Klopotowska JE, de Keizer NF, Van Marum R, Van der Sijs H, de Lange DW, de Jonge E, Abu-Hanna A, Dongelmans DA, Hendriks S, Ten Cate J. Improving medication safety in the Intensive Care by identifying relevant drug-drug interactions-Results of a multicenter Delphi study. Journal of critical care. 2020;57:134-40.

7 Oliveira PC, Silveira MR, Ceccato MD, Reis AM, Pinto IV, Reis EA. Prevalência e Fatores Associados à Polifarmácia em Idosos Atendidos na Atenção Primária à Saúde em Belo Horizonte-MG, Ciência \& Saúde Coletiva. 2021;26:1553-64.

8 Magalhães AC, Cantanhede AM, Drummond BM, Drumond YA, Miranda VF. Avaliação da implantação do serviço de farmácia clínica na Unidade de Terapia Intensiva para contribuir na segurança do paciente. Rev. Med. Minas Gerais. 2016;26(5):16-22.

9 SESAB, Secretaria Estadual de Saúde da Bahia. Protocolo Estadual de Classificação de Risco/ SESAB. Salvador: Secretaria de Saúde do Estado da Bahia; 2014.

10 Oliveira MV, Buarque DC. Polifarmácia e medicamentos potencialmente inapropriados em idosos admitidos em um hospital terciário. Geriatr. Gerontol. Aging. 2018;38-44.

11 Micromedex. IBM Micromedex Drug Ref. Copyright IBM Corporation; 2018.

12 WHO. World Health Organization. Collaborating Centre for DrugStatisticsMethodology. Guidelines for ATC classificationand DDD assignment. Oslo: WHO Collaborating Centre for Drug Statistics Methodology. 2020. Disponível em: https://www.whocc.no/atc_ddd_index/.

13 Silva UDA, Soeiro CLS, Resque RL, Gomes MRF, Costa ÉRG, Fujishima MAT, et al. Interações medicamentosas e consequentes intervenções farmacêuticas na Unidade de Terapia Intensiva de um hospital privado em Macapá, Amapá. Vigilância Sanitária em Debate. 2018;6(2):29-37.

14 Moreira Maiara Benevides, Mesquita Maria Gefé da Rosa, Stipp Marluci Andrade Conceição, Paes Graciele Oroski. Potential intravenous drug interactions in intensive care. Rev. esc. enferm. 2017; 51: e03233. 


\section{RECIMA21 - REVISTA CIENTÍFICA MULTIDISCIPLINAR ISSN 2675-6218}

INTERAÇÕES MEDICAMENTOSAS POTENCIAIS E POLIFARMÁCIA EM PRESCRIÇÕES DE PACIENTES ACOMPANHADOS POR FARMACÊUTICOS CLÍNICOS EM UNIDADES DE TERAPIA INTENSIVA Beatriz da Silva Santos, Tamiles Daiane Borges Santana, Ana Mércia Silva Mascarenhas, Manoela dos Santos Silva, Alaine Azevedo Barbosa, Bianca Oliveira Souza, Danilo Bomfim Miranda, Dara Evinny Santos de Oliveira, Gisele da Silveira Lemos

15 Silva JS, Damascena RS. Avaliação das interações medicamentosas potenciais no âmbito da UTI adulta. Revista de Psicologia. 2017;12(39):1-24.

16 Amaral RO. Prevalência das interações medicamentosas clinicamente manifestadas em pacientes hospitalizados: uma revisão sistemática [Monografia] Departamento de Farmácia, Centro de Ciências Biológicas e da Saúde, Universidade Federal de Sergipe, São Cristóvão; 2016.

17 Rodrigues AT, Stahlschmidt R, Granja S, Pilger D, Falcão ALE, Mazzola PG. Prevalência de potenciais interações medicamentosas na unidade de terapia intensiva de um hospital universitário brasileiro. Braz. J. Pharm. Sci. 2017;53(1):e16109.

18 Alves BL, Silva VG, Caetano IB, Livinalli A, Cruz ML. Polimedicação em Idosos Submetidos a Tratamento Oncológico. Revista Brasileira de Cancerologia. 2019;65(4).

19 Sousa AB, Oliveira JD, Cavalcante AL, Nobre CA, Melo OF, Siqueira RM. Análise de interações medicamentosas potenciais em pacientes de unidade de terapia intensiva de um hospital de ensino de Sobral. Revista Eletrônica Acervo Saúde. 2019;3(17):e320.

20 Dias BM, Santos FS, Reis AM. Potential drug interactions in drug therapy prescribed for older adults at hospital discharge: cross-sectional study. Sao Paulo Medical Journal. 2019;137(4):369-78.

21 Júnior OD, Gasparino RC. Drogas vasoativas: conhecimento da equipe de enfermagem. Revista Baiana de Enfermagem. 2017;31(2).

22 Guzmán-Terán C. Análisis de usos y resistencia a antibióticos en una UCl de Montería, Colombia. Rev. Méd. Risaralda. 2018;24.

23 Silva TMF, Filho AM, Sousa ZS, Rodrigues JAL, Silva NVF, Filho MMF, et al. Infecções hospitalares associadas à bacilos gram-negativos não fermentadores em unidade de terapia intensiva: revisão narrativa. Revista Eletrônica Acervo Saúde. 2021;13(3):e6685.

24 Marsilio NR, Silva DD, Bueno D. Incompatibilidades medicamentosas em centro de tratamento intensivo adulto de um hospital universitário. Revista brasileira de terapia intensiva. 2016;28(2):147-53.

25 Neta AB, Chaves AC, Dias LR, Mascarenhas IR, Macêdo IM, Guizzetti MI, Lacerda TF, de Sousa Machado LC. Relações da corticoterapia no tratamento do choque séptico. Brazilian Journal of Health Review. 2020;3(1):1324-30.

26 Garske CC, Brixner B, Freitas AP, Schneider AP. Avaliação das interações medicamentosas potenciais em prescrições de pacientes em unidade de terapia intensiva. Saúde e Pesquisa. 2016;9(3):483-90.

27 Gomes AM, Bezerra KG, de Sousa Oliveira F. Avaliação de potenciais interações medicamentosas em pacientes da unidade de terapia intensiva de um hospital universitário. Revista de Ciências Médicas e Biológicas. 2019;18(2):183-9.

28 Fentanyl \& Ranitidine. In Micromedex: Drug Interactions para Android (versão v2310) [software de aplicativo móvel]. Greenwood Village (CO): IBM Corporation; 2018. Available from: http://play.google.com.

29 Piperacillin + Tazobactam \& Vancomycin. In Micromedex: Drug Interactions para Android (versão v2310) [software de aplicativo móvel]. Greenwood Village (CO): IBM Corporation; 2018. Available from: http://play.google.com.

30 Alvarez MCM. Nephrotoxicity of antimicrobials and antibiotics. Advances in chronic kidney disease. 2020;27(1):31-7. 


\section{RECIMA21 - REVISTA CIENTÍFICA MULTIDISCIPLINAR ISSN 2675-6218}

INTERAÇÕES MEDICAMENTOSAS POTENCIAIS E POLIFARMÁCIA EM PRESCRIÇÕES DE PACIENTES ACOMPANHADOS POR FARMACÊUTICOS CLÍNICOS EM UNIDADES DE TERAPIA INTENSIVA Beatriz da Silva Santos, Tamiles Daiane Borges Santana, Ana Mércia Silva Mascarenhas, Manoela dos Santos Silva, Alaine Azevedo Barbosa, Bianca Oliveira Souza, Danilo Bomfim Miranda, Dara Evinny Santos de Oliveira, Gisele da Silveira Lemos

31 Phenytoin \& Ranitidine. In Micromedex: Drug Interactions para Android (versão v2310) [software de aplicativo móvel]. Greenwood Village (CO): IBM Corporation; 2018. Available from: http://play.google.com.

32 Silva LD, Matos GC, Barreto BG, Albuquerque DC. Aprazamento de medicamentos por enfermeiros em prescrições de hospital sentinela. Texto \& Contexto-Enfermagem. 2013;22(3):722-30.

33 Fentanyl \& Phenytoin. In Micromedex: Drug Interactions para Android (versão v2310) [software de aplicativo móvel]. Greenwood Village (CO): IBM Corporation; 2018. Available from: http://play.google.com.

34 Phenytoin \& Midazolam. In Micromedex: Drug Interactions para Android (versão v2310) [software de aplicativo móvel]. Greenwood Village (CO): IBM Corporation; 2018. Available from: http://play.google.com.

35 Clopidogrel \& Simvastatin. In Micromedex: Drug Interactions para Android (versão v2310) [software de aplicativo móvel]. Greenwood Village (CO): IBM Corporation; 2018. Available from: http://play.google.com.

36 Yunes LP, Coelho TD, de Almeida SM. Principais interações medicamentosas em pacientes da UTI-adulto de um hospital privado de Minas Gerais. Revista Brasileira de Farmácia Hospitalar e Serviços de Saúde. 2011; 2(3).

37 Fentanyl \& Prednisone. Micromedex: Drug Interactions para Android (versão v2310) [software de aplicativo móvel]. Greenwood Village (CO): IBM Corporation; 2018. Available from: http://play.google.com.

38 Johansen ET, Haustreis SM, Mowinckel AS, Ytrebo LM. Effects of implementing a clinical pharmacist service in a mixed Norwegian ICU. Eur J Hosp Pharm. 2016;23:197-202.

39 Cortes AL, Silvino ZR. Fatores associados a interações Medicamentosas Potenciais em um Centro de Terapia Intensiva: estudo transversal.Escola Anna Nery. 2019;23(3).

40 Pinto MCB. Interações medicamentosas relevantes no tratamento de doenças cardiovasculares [Dissertação]. Departamento de Ciências Farmacêuticas Universidade Fernando Pessoa; 2014.

41 Anvisa. Resolução № 3.259, De 26 De Agosto De 2020, que determina a proibição da comercialização, distribuição, fabricação, importação, manipulação e propaganda do cloridrato de ranitidina, de forma definitiva. Diário Oficial da União. Brasília (DF); 2020; Ed. 165; S. 1; Pág. 164.

42 Veras KN. Condutas Práticas para Unidades de Terapia Intensiva. 2019. Disponível em: http://www.amape.com.br/wp-content/uploads/2019/04/SJT-Condutas-pra ticas-em-UTI.pdf. Acesso em: 18 maio 2021.

43 Reis MA, Gabriel CS, Zanetti AC, Bernardes A, Laus AM, Pereira LR. Medicamentos potencialmente perigosos: identificação de riscos e barreiras de prevenção de erros em terapia intensiva. Texto \& Contexto-Enfermagem. 2018;27(2).

44 Fernandes LL. A importância do farmacêutico hospitalar juntamente com a equipe multidisciplinar na Unidade de Terapia Intensiva (UTI). Revista Farol. 2019;8(8):5-21.

45 Magalhães AC, Cantanhede AM, Drummond BM, Drumond YA, Miranda VF. Avaliação da implantação do serviço de farmácia clínica na Unidade de Terapia Intensiva para contribuir na segurança do paciente. Rev Med Minas Gerais. 2016;26(5):16-22. 